\title{
ON THE SCHRÖDINGER-POISSON-SLATER SYSTEM: BEHAVIOR OF MINIMIZERS, RADIAL AND NONRADIAL CASES
}

\author{
DAVID RUIZ
}

\begin{abstract}
This paper is motivated by the study of a version of the so-called Schrödinger-Poisson-Slater problem:

$$
-\Delta u+\omega u+\lambda\left(u^{2} \star \frac{1}{|x|}\right) u=|u|^{p-2} u,
$$

where $u \in H^{1}\left(\mathbb{R}^{3}\right)$. We are concerned mostly with $p \in(2,3)$. The behavior of radial minimizers motivates the study of the static case $\omega=0$. Among other things, we obtain a general lower bound for the Coulomb energy, that could be useful in other frameworks. The radial and nonradial cases turn out to yield essentially different situations.
\end{abstract}

\section{INTRODUCTION}

Our starting point is the system of Hartree-Fock equations: (1)

$$
-\Delta \psi_{k}+\left(V(x)-E_{k}\right) \psi_{k}+\psi_{k}(x) \int_{\mathbb{R}^{3}} \frac{|\rho(y)|^{2}}{|x-y|} d y-\sum_{j=1}^{N} \psi_{j}(x) \int_{\mathbb{R}^{3}} \frac{\overline{\psi_{j}(y)} \psi_{k}(y)}{|x-y|} d y=0,
$$

where $\psi_{k}: \mathbb{R}^{3} \rightarrow \mathbb{C}$ form an orthogonal set in $H^{1}, \rho=\frac{1}{N} \sum_{j=1}^{N}\left|\psi_{j}\right|^{2}, V(x)$ is an exterior potential and $E_{k} \in \mathbb{R}$. This system appeared in Quantum Mechanics in the study of a system of $N$ particles. With respect to the Hartree equations, it has the advantage of being consistent with the Pauli exclusion principle.

In (1), the last term is usually called the exchange term, and is the most difficult term to be treated. A very simple approximation of this term was given by Slater [27] in the form:

$$
\sum_{j=1}^{N} \psi_{j} \int_{\mathbb{R}^{3}} \frac{\overline{\psi_{j}(y)} \psi_{k}(y)}{|x-y|} d y \sim C_{s} \rho^{1 / 3} \psi_{k}
$$

where $C_{s}$ is a positive constant.

Key words and phrases. Schrödinger-Poisson-Slater equation, Coulomb energy.

The author has been supported by the Spanish Ministry of Science and Technology under Grant MTM2005-01331 and by J. Andalucía (FQM 116). 
By a mean field approximation, the local density $\rho$ can be estimated as $\rho=|u|^{2}$, where $u$ is a solution of the problem:

$$
-\Delta u(x)+V(x) u(x)+B u(x) \int_{\mathbb{R}^{3}} \frac{|u(y)|^{2}}{|x-y|} d y=C|u(x)|^{2 / 3} u(x) .
$$

This system receives the name of Schrödinger-Poisson-Slater system (see [3, 4, 5, 22]).

In this paper we are interested in the following version of the SchrödingerPoisson-Slater problem:

$$
-\Delta u+u+\lambda\left(u^{2} \star \frac{1}{|x|}\right) u=|u|^{p-2} u
$$

where $\lambda>0$. We are concerned with the case $p \in(2,3)$, and we mainly consider positive solutions. The case $p \geq 3$ is different and has been studied in [2, 18, 25].

In recent years problem (2) has been object of intensive research, see $[2,11,12,13,14,17,18,[19,23,24,25,26,31$. We point out that (2) presents a combination of repulsive forces (given by the nonlocal term) and attractive forces (given by the power term). As we shall see, the interaction between them gives rise to non expected situations.

The associated energy functional is $I_{\lambda}: H^{1}\left(\mathbb{R}^{3}\right) \rightarrow \mathbb{R}$,

$$
I_{\lambda}(u)=\frac{1}{2} \int_{\mathbb{R}^{3}}\left(|\nabla u|^{2}+u^{2}\right) d x+\frac{\lambda}{4} \int_{\mathbb{R}^{3}} \int_{\mathbb{R}^{3}} \frac{u^{2}(x) u^{2}(y)}{|x-y|} d x d y-\frac{1}{p} \int_{\mathbb{R}^{3}}|u|^{p} d x .
$$

The original motivation of this paper is the following. In [25] it is shown that $\left.I_{\lambda}\right|_{H_{r}^{1}}$ is bounded below for any positive value of $\lambda$, where $H_{r}^{1}$ denotes the Sobolev space of radial functions. Moreover, when $\lambda$ is small, there exist nontrivial radial minimizers that blow up as $\lambda \rightarrow 0$.

One could ask how is the profile of those solutions as $\lambda \rightarrow 0$. A partial answer is given in [13, 24. In those papers, by using a perturbation technique, solutions of (2) with a certain behavior are found (for $\lambda$ small). Moreover, those solutions correspond to local minima of $\left.I_{\lambda}\right|_{H_{r}^{1}}$ and their energy tend to $-\infty$ as $\lambda \rightarrow 0$, so it is quite reasonable to think that those solutions correspond to global minima. However, those solutions are provided only if $p<18 / 7$. This exponent appears also in more recent work on concentration on spheres, see [17].

At this point, some natural questions arise: what is the meaning of the value $p=18 / 7$ ? How do minimizers behave if $p \in(18 / 7,3)$ ? Observe that the most important case in applications, $p=8 / 3$, belongs to this interval. In this paper we find answers to both questions.

By making the change of variables $v(x)=\varepsilon^{\frac{2}{p-2}} u(\varepsilon x), \varepsilon=\lambda^{\frac{p-2}{4(3-p)}}$, we arrive to the problem:

$$
-\Delta v+\varepsilon^{2} v+\left(v^{2} \star \frac{1}{|x|}\right) v=|v|^{p-2} v
$$


This motivates the study of the limit problem:

$$
-\Delta v+\left(v^{2} \star \frac{1}{|x|}\right) v=|v|^{p-2} v .
$$

Problem (4) can be thought of as a zero mass problem (see [7]), but under the action of a nonlocal term. To start with, $H^{1}\left(\mathbb{R}^{3}\right)$ is not the right space to study it. It seems quite clear that the right space should be:

$$
E=E\left(\mathbb{R}^{3}\right)=\left\{u \in D^{1,2}\left(\mathbb{R}^{3}\right): \int_{\mathbb{R}^{3}} \int_{\mathbb{R}^{3}} \frac{u^{2}(x) u^{2}(y)}{|x-y|} d x d y<+\infty\right\} .
$$

The double integral expression is the so-called Coulomb energy of the wave, and has been very studied, see for instance [20]. In other words, $E\left(\mathbb{R}^{3}\right)$ is the space of functions in $D^{1,2}\left(\mathbb{R}^{3}\right)$ such that the Coulomb energy of the charge is finite. We also denote $E_{r}=E\left(\mathbb{R}^{3}\right)$ the subspace of radial functions.

One of the main goals of this paper is the following general inequality:

Theorem 1.1. Given $\alpha>1 / 2$, there exists $c=c(\alpha)>0$ such that for any $u: \mathbb{R}^{N} \rightarrow \mathbb{R}$ measurable function, we have:

$$
\int_{\mathbb{R}^{N}} \int_{\mathbb{R}^{N}} \frac{u^{2}(x) u^{2}(y)}{|x-y|^{N-2}} d x d y \geq c\left(\int_{\mathbb{R}^{N}} \frac{u(x)^{2}}{|x|^{\frac{N-2}{2}}(1+|\log | x||)^{\alpha}} d x\right)^{2} .
$$

In particular, $E \subset L^{2}\left(\mathbb{R}^{3},|x|^{-\frac{1}{2}}(1+|\log | x||)^{-\alpha} d x\right)$ continuously.

We are not aware of any lower bound for the Coulomb energy in this fashion. We think that this inequality can be very useful in other frameworks, such as the Hartree equation or the Thomas-Fermi-Von Weizsäcker model (see [8, 9]). We also show that Theorem 1.1 is "almost sharp": in the right term, the exponent $\frac{N-2}{2}$ is optimal and a logarithmic factor is needed, see Remark 3.3 .

By combining inequality (5) with the results of [29, 30], we obtain the following result, that shows the significance of the exponent $18 / 7$ in the radial case.

Theorem 1.2. $E_{r}\left(\mathbb{R}^{3}\right) \subset L^{p}\left(\mathbb{R}^{3}\right)$ continuously for $p \in\left(\frac{18}{7}, 6\right]$, and the inclusion is compact for $p \in\left(\frac{18}{7}, 6\right)$. Moreover, $E_{r}\left(\mathbb{R}^{3}\right)$ is not included in $L^{p}\left(\mathbb{R}^{3}\right)$ for $p<\frac{18}{7}$ or $p>6$.

With this result in hand, we obtain that for $p \in\left(\frac{18}{7}, 6\right]$, the functional $J: E_{r} \rightarrow \mathbb{R}$,

$$
J(v)=\frac{1}{2} \int_{\mathbb{R}^{3}}|\nabla v|^{2} d x+\frac{1}{4} \int_{\mathbb{R}^{3}} \int_{\mathbb{R}^{3}} \frac{v^{2}(x) v^{2}(y)}{|x-y|} d x d y-\frac{1}{p} \int_{\mathbb{R}^{3}}|v|^{p} d x,
$$

is well-defined, $C^{1}$, and its critical points correspond to solutions of (4). Moreover:

Theorem 1.3. For any $p \in(18 / 7,3), J$ is coercive and weak lower semicontinuous. Therefore, it attains its infimum, which is negative. As a consequence, (4) has a positive solution in $E$. 
We do not think that the above solution belongs to $L^{2}\left(\mathbb{R}^{3}\right)$, so it does not correspond to a real physical situation. However, it can be used to describe the asymptotic behavior of the minimizers of $I_{\lambda}$ :

Theorem 1.4. Suppose that $p \in(18 / 7,3)$ and let $u_{\lambda}$ be a minimizer of $\left.I_{\lambda}\right|_{H_{r}^{1}}$. Then, as $\lambda \rightarrow 0$,

$$
u_{\lambda}=\varepsilon^{-\frac{2}{p-2}} v_{\varepsilon}\left(\frac{x}{\varepsilon}\right)
$$

where $\varepsilon=\lambda^{\frac{p-2}{4(3-p)}}$ and $d\left(v_{\varepsilon}, K\right) \rightarrow 0$. Here $K \subset E$ is the set of minimizers:

$$
K=\{v \in E: J(v)=\min J\},
$$

and $d(v, K)=\inf \left\{\|v-w\|_{E}: w \in K\right\}$. In particular, given $\lambda_{n} \rightarrow 0$, we have that $\varepsilon_{n} \rightarrow 0$ and $v_{\varepsilon_{n}} \rightarrow v$ in $E$ (up to a subsequence) where $v$ is a minimizer of $J$.

We point out that, as $\lambda \rightarrow 0$, radial minimizers behave differently depending on $p$. For $p>18 / 7$ minimizers tend to concentrate around zero and blow up in $L^{\infty}$ norm. On the other hand, for $p<18 / 7$, it is reasonable to think that the solutions given in [13, 24] are minimizers; those solutions spread out and are bounded in $L^{\infty}\left(\mathbb{R}^{3}\right)$.

So far, we have always considered the radial case. The last section of the paper is devoted to investigate the nonradial case. We point out that the situation in both cases turns out to be very different.

To start with, we have the following result (to be compared with Theorem 1.2):

Theorem 1.5. $E\left(\mathbb{R}^{3}\right) \subset L^{p}\left(\mathbb{R}^{3}\right)$ for any $p \in[3,6)$, and the inclusion does not hold for $p<3$.

Moreover, from [25] we know that if $p \in(2,3),\left.I_{\lambda}\right|_{H_{r}^{1}}$ is always bounded below and attains its infimum. It is also easy to prove that the map $\lambda \mapsto$ $\left.\inf I_{\lambda}\right|_{H_{r}^{1}}$ is continuous and tends to $-\infty$ as $\lambda \rightarrow 0$. However:

Theorem 1.6. If $p \in(2,3)$, there exists $\lambda_{0}>0$ such that inf $I_{\lambda}=0$ for $\lambda \geq \lambda_{0}$ and $\inf I_{\lambda}=-\infty$ for $\lambda<\lambda_{0}$.

As we see, the nonlocal term leads to different situations in the radial and nonradial cases. In order to study in depth this phenomenon, we consider the problem in a ball:

$$
\begin{array}{ll}
-\Delta u+u+\lambda\left(u^{2} \star \frac{1}{|x|}\right) u=|u|^{p-2} u, & \text { in } B(0, R) \\
u(x)=0 & \text { in } \partial B(0, R) .
\end{array}
$$

In the following theorem we obtain a result of breaking of symmetry of minimizers:

Theorem 1.7. Suppose that either:

(1) $p \in(2,3), \lambda \in\left(0, \lambda_{0}\right)$ and $R$ large enough, 
or

(2) $p \in(18 / 7,3), \lambda$ small.

Then, inf $\left.I_{\lambda}\right|_{H_{0}^{1}(B(0, R))}$ is attained at a nonradial function.

Observe that here the well-known Gidas-Ni-Nirenberg result ([15]) does not hold (because of the nonlocal term). Nonradial ground states have been found in other frameworks previously, like in the Hénon equation, see 28. However, observe that in our case it is a free minimizer of the energy functional, not a minimizer under a certain constraint. In particular, it is an orbitally stable solution in the sense of [10] (see also Remark 5.3).

The paper is organized as follows. In Section 2 we establish some notations and we present a preliminary study of the space $E$. In Section 3 we prove Theorem 1.1, which provides us with a general inequality that will be used in the following section. Section 4 is devoted to the radial case; we prove Theorems 1.2, 1.3, and 1.4. In Section 5 we deal with the nonradial case, and prove Theorems $1.5,1.6$ and 1.7 .

\section{Preliminaries}

In this section we establish some notation that will be used throughout the paper. We also define the space $E$ and study some basic properties of it.

We will use the following common notations:

- $C_{0}^{\infty}\left(\mathbb{R}^{N}\right)$ is the set of $C^{\infty}$ functions with compact support.

- $H^{1}\left(\mathbb{R}^{3}\right)=\left\{u \in L^{2}\left(\mathbb{R}^{3}\right):|\nabla u| \in L^{2}\left(\mathbb{R}^{3}\right)\right\}$ is the usual Sobolev space, and $\|\cdot\|_{H^{1}}$ denotes its norm.

- $D^{1,2}\left(\mathbb{R}^{N}\right)=\left\{u \in L^{\frac{2 N}{N-2}}\left(\mathbb{R}^{N}\right):|\nabla u| \in L^{2}\left(\mathbb{R}^{N}\right)\right\}$, with the usual norm $\|u\|_{D}=\|\nabla u\|_{L^{2}}$.

- Given any $\Omega \subset \mathbb{R}^{N}$ a smooth domain, we denote by $H_{0}^{1}(\Omega)$ as the completion of $C_{0}^{\infty}(\Omega)$ with the $\|\cdot\|_{H^{1}}$ norm.

- We write $C_{0, r}^{\infty}\left(\mathbb{R}^{N}\right), H_{r}^{1}\left(\mathbb{R}^{N}\right), D_{r}^{1,2}\left(\mathbb{R}^{N}\right), H_{0, r}^{1}(B(0, R))$ to denote the corresponding subspaces of radial functions.

Definition 2.1. We define the space $E$ :

$$
E=E\left(\mathbb{R}^{N}\right)=\left\{u \in D^{1,2}\left(\mathbb{R}^{N}\right): \int_{\mathbb{R}^{N}} \int_{\mathbb{R}^{N}} \frac{u^{2}(x) u^{2}(y)}{|x-y|^{N-2}} d x d y<+\infty\right\}
$$

That is, $E\left(\mathbb{R}^{N}\right)$ is the space of functions in $D^{1,2}\left(\mathbb{R}^{N}\right)$ such that the Coulomb energy of the charge is finite. We denote by $E_{r}=E_{r}\left(\mathbb{R}^{N}\right)$ to the subspace of radial functions.

We begin by studying some elementary properties of $E$ :

Proposition 2.2. Let us define, for any $u \in E$,

$$
\|u\|_{E}=\left(\int_{\mathbb{R}^{N}}|\nabla u(x)|^{2} d x+\left(\int_{\mathbb{R}^{N}} \int_{\mathbb{R}^{N}} \frac{u^{2}(x) u^{2}(y)}{|x-y|^{N-2}} d x d y\right)^{1 / 2}\right)^{1 / 2} .
$$


Then, $\|\cdot\|_{E}$ is a norm, and $\left(E,\|\cdot\|_{E}\right)$ is a uniformly convex Banach space. Moreover, $C_{0}^{\infty}\left(\mathbb{R}^{N}\right)$ is dense in $E$, and also $C_{0, r}^{\infty}\left(\mathbb{R}^{N}\right)$ is dense in $E_{r}$.

Proof. We will need the following general result, that must be well-known. Its proof is elementary and will be skipped.

Lemma 2.3. Let $X$ be a vectorial space, $p, q$ two uniformly convex norms on $X$, and $\|x\|=\sqrt{p(x)^{2}+q(x)^{2}}$. Then $\|\cdot\|$ is also a uniformly convex norm.

As usually, (see [8, 9, 20]), let us define

$$
D(f, g)=\int_{\mathbb{R}^{N}} \int_{\mathbb{R}^{N}} \frac{f(x) g(y)}{|x-y|^{N-2}} d x d y .
$$

The inequality $D(f, g)^{2} \leq D(f, f) D(g, g)$ is well-known, see [20], page 214 .

We now show that $\|\cdot\|_{E}$ is a uniformly convex norm. By Lemma 2.3, it suffices to deal with $T(u)=D\left(u^{2}, u^{2}\right)^{1 / 4}$. First, we show that it satisfies the triangular inequality. Actually,

$D\left((u+v)^{2},(u+v)^{2}\right)=D\left(u^{2}, u^{2}\right)+D\left(v^{2}, v^{2}\right)+4 D\left(u^{2}, u v\right)+4 D\left(v^{2}, u v\right)+4 D(u v, u v)+2 D\left(u^{2}, v^{2}\right)$.

We now estimate:

$$
D\left(u^{2}, v^{2}\right) \leq \sqrt{D\left(u^{2}, u^{2}\right) D\left(v^{2}, v^{2}\right)} .
$$

In the next computation, we just use Hölder inequality:

$$
D(u v, u v)=\int_{\mathbb{R}^{N}} \int_{\mathbb{R}^{N}} \frac{u(x) v(x) u(y) v(y)}{|x-y|^{N-2}} d x d y \leq \sqrt{D\left(u^{2}, u^{2}\right) D\left(v^{2}, v^{2}\right)} .
$$

Moreover:

$$
D\left(u^{2}, u v\right) \leq\left(D\left(u^{2}, u^{2}\right) D(u v, u v)\right)^{1 / 2} \leq\left(D\left(u^{2}, u^{2}\right) \sqrt{D\left(u^{2}, u^{2}\right) D\left(v^{2}, v^{2}\right)}\right)^{1 / 2} .
$$

An analogous estimate works for $D\left(v^{2}, u v\right)$. Putting all estimates together, we obtain $T(u+v) \leq T(u)+T(v)$.

With respect to uniform convexity, we can argue as before to obtain the following inequality: for any $u, v \in E$,

$$
T\left(\frac{u+v}{2}\right)^{4}+T\left(\frac{u-v}{2}\right)^{4} \leq \frac{T(u)^{4}+T(v)^{4}}{2} .
$$

This readily implies that $T$ is uniformly convex.

In order to show that $E$ is a Banach space take a Cauchy sequence $u_{n}$ in $E$. Clearly, $u_{n}$ is a Cauchy sequence in $D^{1,2}$; we now show that $\frac{u_{n}(x) u_{n}(y)}{|x-y|^{\frac{N-2}{2}}}$ is also a Cauchy sequence in $L^{2}\left(\mathbb{R}^{2 N}\right)$. Indeed:

$$
\begin{gathered}
\int_{\mathbb{R}^{N}} \int_{\mathbb{R}^{N}} \frac{(u(x) u(y)-v(x) v(y))^{2}}{|x-y|^{N-2}} d x d y= \\
\int_{\mathbb{R}^{N}} \int_{\mathbb{R}^{N}} \frac{(u(x) u(y)-u(x) v(y)+u(x) v(y)-v(x) v(y))^{2}}{|x-y|^{N-2}} d x d y \leq
\end{gathered}
$$




$$
\begin{gathered}
2 \int_{\mathbb{R}^{N}} \int_{\mathbb{R}^{N}} \frac{u(x)^{2}(u(y)-v(y))^{2}+v(y)^{2}(u(x)-v(x))^{2}}{|x-y|^{N-2}} d x d y= \\
2 \int_{\mathbb{R}^{N}} \int_{\mathbb{R}^{N}} \frac{\left(u(x)^{2}+v(x)^{2}\right)(u(y)-v(y))^{2}}{|x-y|^{N-2}} d x d y=2 D\left(u^{2}+v^{2},(u-v)^{2}\right) \leq \\
2 \sqrt{D\left(u^{2}+v^{2}, u^{2}+v^{2}\right) D\left((u-v)^{2},(u-v)^{2}\right)} .
\end{gathered}
$$

So, $u_{n} \rightarrow u$ in $D^{1,2}$ and $\frac{u_{n}(x) u_{n}(y)}{|x-y|^{\frac{N-2}{2}}} \rightarrow \psi(x, y)$ in $L^{2}$. Passing to a subsequence and by uniqueness of pointwise convergence we conclude that $\frac{u(x) u(y)}{|x-y|^{\frac{N-2}{2}}}=\psi(x, y)$.

Finally, observe that $C_{0}^{\infty}\left(\mathbb{R}^{N}\right)$ is dense in $H^{1}\left(\mathbb{R}^{N}\right)$, and hence $\overline{C_{0}^{\infty}\left(\mathbb{R}^{N}\right)}$ ' $H^{1}\left(\mathbb{R}^{N}\right)$. So, it suffices to show that $H^{1}\left(\mathbb{R}^{N}\right)$ is dense. Take $u \in E$, and choose $\xi \in C_{0, r}^{\infty}\left(\mathbb{R}^{N}\right), \xi(x)=1$ for $x \in B(0,1)$. It follows easily that $u_{n}(x)=\xi\left(\frac{x}{n}\right) u(x)$ belongs to $H^{1}\left(\mathbb{R}^{N}\right)$ and $u_{n} \rightarrow u$ in $E$.

Analogously we can argue for the radial case.

Let us define $\phi_{u}=\frac{1}{4 \pi|x|^{N-2}} \star u^{2}$; then, $u \in E$ if and only if both $u$ and $\phi_{u}$ belong to $D^{1,2}\left(\mathbb{R}^{N}\right)$. In such case, $-\Delta \phi_{u}=u^{2}$ in a weak sense, and

$$
\int_{\mathbb{R}^{N}}\left|\nabla \phi_{u}(x)\right|^{2} d x=\int_{\mathbb{R}^{N}} \phi_{u}(x) u(x)^{2} d x=\int_{\mathbb{R}^{N}} \int_{\mathbb{R}^{N}} \frac{u^{2}(x) u^{2}(y)}{4 \pi|x-y|^{N-2}} d x d y .
$$

Proposition 2.4. Given a sequence $\left\{u_{n}\right\}$ in $E, u_{n} \rightarrow u$ in $E$ if and only if $u_{n} \rightarrow u$ in $D^{1,2}$ and $\int_{\mathbb{R}^{N}} \int_{\mathbb{R}^{N}} \frac{u_{n}^{2}(x) u_{n}^{2}(y)}{|x-y|^{N-2}} d x d y$ is bounded. In such case, $\phi_{n} \rightarrow \phi_{u}$ in $D^{1,2}$, where $\phi_{n}=\phi_{u_{n}}$.

Proof. Clearly, the implication to the right is obvious. Suppose now that $u_{n} \rightarrow u$ in $D^{1,2}\left(\mathbb{R}^{N}\right)$ and $\int_{\mathbb{R}^{N}} \int_{\mathbb{R}^{N}} \frac{u_{n}^{2}(x) u_{n}^{2}(y)}{|x-y|^{N-2}} d x d y$ is bounded. In particular, $\left\|u_{n}\right\|_{E}$ is bounded.

Suppose, reasoning by contradiction, that $u_{n}$ does not converge weakly to $u$ in $E$. So, there exists a neighborhood of $u$ in the weak topology and a subsequence (still denoted by $u_{n}$ ) such that $u_{n} \notin V$. Being $E$ uniformly convex, it is reflexive (see [6], Theorem III.29), and hence $u_{n} \rightarrow v$ in $E$ (up to another subsequence) for some $v \in E$. But this implies that $u_{n} \rightarrow v$ in $D^{1,2}\left(\mathbb{R}^{N}\right)$, and by uniqueness, $v=u$. This contradicts $u_{n} \notin V$.

In order to prove that $\phi_{n} \rightarrow \phi_{u}$ in $D^{1,2}$, observe that $\phi_{n}$ is bounded in $D^{1,2}\left(\mathbb{R}^{N}\right.$ ), and therefore $\phi_{n} \rightarrow \phi$ in $D^{1,2}$ for some $\phi$ (up to a subsequence but, again, this suffices).

Take any $\rho \in C_{0}^{\infty}\left(\mathbb{R}^{N}\right)$ and compute:

$$
\int_{\mathbb{R}^{N}} \nabla \phi \cdot \nabla \rho \leftarrow \int_{\mathbb{R}^{N}} \nabla \phi_{n} \cdot \nabla \rho=\int_{\mathbb{R}^{N}} u_{n}^{2} \rho \rightarrow \int_{\mathbb{R}^{N}} u^{2} \rho .
$$

The last convergence follows from the fact that $\left.\left.\left(u_{n}\right)\right|_{K} \rightarrow u\right|_{K}$ strongly in $L^{2}$ for any compact set $K$. Hence, $-\Delta \phi=u^{2}$, that is, $\phi=\phi_{u}$. 


\section{A LOWER Bound of the Coulomb EnERGy}

In this section we study some bounds of the Coulomb energy, that will be of use later on. In particular, we prove Theorem 1.1, in which a lower bound of the Coulomb energy is given.

First, by using Hardy-Littlewood-Sobolev inequality (see [20], page 98), we have the following bound on the Coulomb energy:

$$
\int_{\mathbb{R}^{N}} \int_{\mathbb{R}^{N}} \frac{u^{2}(x) u^{2}(y)}{|x-y|^{N-2}} d x d y \leq C\|u\|_{L^{\frac{4 N}{N+2}}}^{4} .
$$

In particular, we have that $D^{1,2}\left(\mathbb{R}^{N}\right) \cap L^{\frac{4 N}{N+2}}\left(\mathbb{R}^{N}\right) \subset E\left(\mathbb{R}^{N}\right)$ continuously.

Let us consider now $E_{r}\left(\mathbb{R}^{N}\right)$ the subspace of $E$ of radially symmetric functions. In such case, also $\phi_{u}$ is a radial function. For this subspace, we obtain another upper bound:

$$
\begin{aligned}
\left\|\phi_{u}\right\|_{D}^{2}= & \int_{\mathbb{R}^{N}}\left|\nabla \phi_{u}(x)\right|^{2} d x=\int_{\mathbb{R}^{N}} \phi_{u}(x) u(x)^{2} d x \leq C\left\|\phi_{u}\right\|_{D} \int_{\mathbb{R}^{N}} u(x)^{2}|x|^{-\frac{N-2}{2}} d x \Rightarrow \\
\text { (8) } \quad & \int_{\mathbb{R}^{N}} \int_{\mathbb{R}^{N}} \frac{u^{2}(x) u^{2}(y)}{|x-y|^{N-2}} d x d y \leq C\left(\int_{\mathbb{R}^{N}} u(x)^{2}|x|^{-\frac{N-2}{2}} d x\right)^{2} .
\end{aligned}
$$

In the estimates above we have used the point-wise estimate:

$$
v(x) \leq C\|v\|_{D}|x|^{-\frac{N-2}{2}} \forall x \neq 0,
$$

for some $C>0$ and for every $v \in D_{r}^{1,2}\left(\mathbb{R}^{N}\right)$. This estimate appears in [7] (page 340) for $|x|>1$, but a rescaling argument implies its validity for any $x \neq 0$. Therefore, we have that $D_{r}^{1,2}\left(\mathbb{R}^{N}\right) \cap L^{2}\left(\mathbb{R}^{N},|x|^{-\frac{N-2}{2}} d x\right) \subset E_{r}$.

We point out that estimate (8) does not hold if $u$ is not radial, as one can easily check by making use of translations.

Both (7) and (8) are upper bounds of the Coulomb energy, which provide us with sufficient conditions for $u$ to belong to $E$. In this section we prove Theorem 1.1 which provides us with a necessary condition, that is, a lower bound. As far as we know, no lower bound of the Coulomb energy has been given in the literature so far.

The main result of this section is the following:

Theorem 3.1. Let $q>0, \alpha>1 / 2$. Then there exists $c=c(q, \alpha)>0$ such that for any $f: \mathbb{R}^{N} \rightarrow[0,+\infty)$ measurable, we have:

$$
\int_{\mathbb{R}^{N}} \int_{\mathbb{R}^{N}} \frac{f(x) f(y)}{|x-y|^{q}} d x d y \geq c\left(\int_{\mathbb{R}^{N}} \frac{f(x)}{|x|^{\frac{q}{2}}(1+|\log | x||)^{\alpha}} d x\right)^{2} .
$$

Observe that Theorem 1.1 follows trivially from the above result.

Proof. We begin by estimating: 


$$
\begin{gathered}
\int_{\mathbb{R}^{N}} \int_{\mathbb{R}^{N}} \frac{f(x) f(y)}{|x-y|^{q}} d x d y \geq \int_{\mathbb{R}^{N}} \int_{|y|<2|x|<4|y|} \frac{f(x) f(y)}{|x|^{q / 2}|y|^{q / 2}} \frac{|x|^{q / 2}|y|^{q / 2}}{|x-y|^{q}} d x d y \geq \\
c(q) \int_{\mathbb{R}^{N}} \int_{|y|<2|x|<4|y|} \frac{f(x) f(y)}{|x|^{q / 2}|y|^{q / 2}} d x d y= \\
c(q) \int_{s=0}^{+\infty} \int_{r=s / 2}^{2 s}\left(\frac{1}{r^{q / 2}} \int_{|x|=r} f(x) d \sigma_{x}\right)\left(\frac{1}{s^{q / 2}} \int_{|y|=s} f(y) d \sigma_{y}\right) d r d s .
\end{gathered}
$$

The rest of the proof is based on the following lemma:

Lemma 3.2. Let $\alpha>1 / 2$; then, there exists $c=c(\alpha)>0$ such that for any $h:(0,+\infty) \rightarrow(0,+\infty)$ measurable function, there holds:

$$
\int_{s=0}^{+\infty} \int_{r=s / 2}^{2 s} h(r)(1+|\log r|)^{\alpha} h(s)(1+|\log s|)^{\alpha} d r d s \geq c\left(\int_{0}^{+\infty} h(r) d r\right)^{2} \text {. }
$$

Indeed, we can define:

$$
h(r)=\frac{1}{r^{q / 2}(1+|\log r|)^{\alpha}} \int_{|x|=r} f(x) d \sigma_{x} .
$$

We now apply the previous lemma and finish the proof of Theorem 3.1.

In order to prove the lemma, the following inequality will be of use: for any two sequences of nonnegative numbers $\left\{a_{n}\right\},\left\{b_{n}\right\}(n \in \mathbb{Z})$, we have:

$$
\left(\sum_{n=-\infty}^{+\infty} a_{n}\right)^{2} \leq\left(\sum_{n=-\infty}^{+\infty} \frac{1}{b_{n}}\right)\left(\sum_{n=-\infty}^{+\infty} b_{n} a_{n}^{2}\right.
$$

This inequality is quite well-known, but we show briefly the proof for convenience of the reader. Below we use the inequality $a b \leq \frac{1}{2}\left(\gamma a^{2}+\gamma^{-1} b^{2}\right)$ :

$$
\begin{gathered}
\left(\sum_{|n| \leq K} a_{n}\right)^{2}=\sum_{|n| \leq K} \sum_{|m| \leq K} a_{n} a_{m} \leq \frac{1}{2} \sum_{|n| \leq K} \sum_{|m| \leq K} \frac{b_{n}}{b_{m}} a_{n}^{2}+\frac{b_{m}}{b_{n}} a_{m}^{2}= \\
\sum_{|n| \leq K} \sum_{|m| \leq K} \frac{b_{n}}{b_{m}} a_{n}^{2}=\left(\sum_{|n| \leq K} \frac{1}{b_{n}}\right)\left(\sum_{|n| \leq K} b_{n} a_{n}^{2}\right) .
\end{gathered}
$$

Take $K \rightarrow+\infty$ and we are done.

Let us take $b_{n}=(1+|n|)^{2 \alpha}$ and $a_{n}=\int_{2^{n}}^{2^{n+1}} h(r) d r$. Then:

$$
\begin{gathered}
\left(\int_{0}^{+\infty} h(r) d r\right)^{2}=\left(\sum_{n=-\infty}^{+\infty} a_{n}\right)^{2} \leq C(\alpha) \sum_{n=-\infty}^{+\infty}(1+|n|)^{2 \alpha} a_{n}^{2}= \\
C(\alpha) \sum_{n=-\infty}^{+\infty}\left((1+|n|)^{\alpha} \int_{2^{n}}^{2^{n+1}} h(r) d r\right)^{2}=
\end{gathered}
$$




$$
C(\alpha) \sum_{n=-\infty}^{+\infty}\left((1+|n|)^{\alpha} \int_{2^{n}}^{2^{n+1}} h(r) d r\right)\left((1+|n|)^{\alpha} \int_{2^{n}}^{2^{n+1}} h(s) d s\right) .
$$

Now, for $n \geq 0$, we have that $r>2^{n}$, hence $n<\log _{2} r=\log _{2}(e) \log r$, so $1+n \leq C(1+\log r)$.

Moreover, if $n<0$, we estimate $2^{n+1}>r \Rightarrow 0 \geq n+1>\log _{2} r=$ $\log _{2}(e) \log r$. We take absolute values and obtain that $|n|-1<\log _{2} e|\log r|$, so $1+|n| \leq C(1+|\log r|)$. Analogously, $1+|n| \leq C(1+|\log s|)$ in any integral term above.

Hence:

$$
\begin{gathered}
\sum_{n=-\infty}^{+\infty}\left((1+|n|)^{\alpha} \int_{2^{n}}^{2^{n+1}} h(r) d r\right)\left((1+|n|)^{\alpha} \int_{2^{n}}^{2^{n+1}} h(s) d s\right) \leq \\
C \sum_{n=-\infty}^{+\infty} \int_{2^{n}}^{2^{n+1}} \int_{2^{n}}^{2^{n+1}} h(r)(1+|\log r|)^{\alpha} h(s)(1+|\log s|)^{\alpha} d r d s \leq \\
C \int_{s=0}^{+\infty} \int_{s / 2}^{2 s} h(r)(1+|\log r|)^{\alpha} h(s)(1+|\log s|)^{\alpha} d r d s .
\end{gathered}
$$

Remark 3.3. Observe that in (5) the exponent $\frac{N-2}{2}$ is the same as in inequality (8) for radial functions. With respect to the logarithmic term, we do not know whether inequality (5) holds for some smaller value of $\alpha$ or not. However, we show now that (5) does not hold if $\alpha<\frac{N-2}{2 N}$. Indeed, by combining (7) with the thesis of Theorem 1.1, we conclude the inclusion:

$$
L^{\frac{4 N}{N+2}}\left(\mathbb{R}^{N}\right) \subset L^{2}\left(\mathbb{R}^{N},|x|^{-\frac{N-2}{2}}(1+|\log | x||)^{-\alpha} d x\right) .
$$

Actually one can check that inclusion directly by using Hölder inequality if $\alpha>\frac{N-2}{2 N}$. But (9) is false for $\alpha<\frac{N-2}{2 N}$, as one can easily check by using the function:

$$
f(x)=\frac{1}{|x|^{\frac{N+2}{4}}(1+|\log | x||)^{\beta}},
$$

where $\frac{N+2}{4 N}<\beta \leq \frac{1-\alpha}{2}$. Hence, the thesis of Theorem 1.1 does not hold with $\alpha<\frac{N-2}{2 N}$.

Observe that the previous argument implies that Lemma 3.2 is not true for any $\alpha<1 / 2$. Indeed, if $\alpha<1 / 2$, we can always choose $N$ large enough so that $\alpha<\frac{N-2}{2 N}$. Observe now that Lemma 3.2 would imply (5), which is false by the previous argument.

Remark 3.4. By using translations, we can obtain the following generalization of Theorem 3.1: 
Let $q>0, \alpha>1 / 2$. Then there exists $c=c(q, \alpha)>0$ such that for any $f: \mathbb{R}^{N} \rightarrow[0,+\infty)$ measurable and any $z \in \mathbb{R}^{N}$, we have:

$$
\int_{\mathbb{R}^{N}} \int_{\mathbb{R}^{N}} \frac{f(x) f(y)}{|x-y|^{q}} d x d y \geq c\left(\int_{\mathbb{R}^{N}} \frac{f(x)}{|x-z|^{\frac{q}{2}}(1+|\log | x-z||)^{\alpha}} d x\right)^{2} .
$$

\section{The RADiAL CASE}

From now on we will restrict ourselves to the case $N=3$, since this is the most interesting case in applications. In this section we are concerned with the radial case, and we will prove Theorems 1.2 , 1.3 and 1.4 .

First of all, if $u$ is a radial function, $\phi_{u}$ is also radial, and can be written as (see [13, 24]):

$$
\phi_{u}(r)=\frac{1}{r} \int_{0}^{+\infty} u^{2}(s) \operatorname{smin}\{r, s\} d s
$$

Therefore,

$$
\left\|\phi_{u}\right\|_{D}^{2}=\int_{\mathbb{R}^{3}} \phi_{u}(|x|) u^{2}(|x|) d x=4 \pi \int_{0}^{+\infty} \int_{0}^{+\infty} u^{2}(r) u^{2}(s) r \operatorname{sinin}\{r, s\} d r d s .
$$

Proof of Theorem 1.2. Take any $\gamma>\frac{1}{2}$, and define $V(x)=(1+|x|)^{-\gamma}$. Following [29, 30], let us define:

$$
H_{r}^{1}\left(\mathbb{R}^{3}, V\right):=D_{r}^{1,2}\left(\mathbb{R}^{3}\right) \cap L^{2}\left(\mathbb{R}^{3}, V(x) d x\right) .
$$

In that space we consider the norm $\|u\|_{H_{V}}^{2}=\int_{\mathbb{R}^{3}}|\nabla u(x)|^{2}+V(x) u^{2}(x) d x$.

By Theorem 1.1 we obtain $E_{r}\left(\mathbb{R}^{3}\right) \subset H_{r}^{1}\left(\mathbb{R}^{3}, V\right)$. The spaces of radial functions $H_{r}^{1}\left(\mathbb{R}^{N}, V\right)$ have been studied in $[29,30]$ : there it is proved that

$$
H_{r}^{1}\left(\mathbb{R}^{3}, V\right) \subset L^{p}\left(\mathbb{R}^{3}\right) \text { for } p \in\left[\frac{2(4+\gamma)}{4-\gamma}, 6\right]
$$

with continuous inclusion. Observe now that fixed $p>\frac{18}{7}$, we can take $\gamma>\frac{1}{2}$ such that $p=\frac{2(4+\gamma)}{4-\gamma}$.

We can now prove the compact inclusions as usually, combining the continuous inclusions with asymptotic estimates. Fix $p \in\left(\frac{18}{7}, 6\right)$ and assume, without loss of generality, that $u_{n} \rightarrow 0$ in $E$. In particular, $u_{n} \rightarrow 0$ in $D^{1,2}$ and $u_{n} \rightarrow 0$ in $L^{q}$ for every $q \in\left(\frac{18}{7}, 6\right]$.

We have the asymptotic estimate $\left|u_{n}(x)\right| \leq C\left\|u_{n}\right\|_{D}|x|^{-1 / 2} \leq C^{\prime}|x|^{-1 / 2}$ (actually, a better estimate can be given, see [29, 30]). Choose $\delta>0$ such that $p-\delta>\frac{18}{7}$. We have:

$$
\int_{B(0, R)^{c}} u_{n}^{p}=\int_{B(0, R)^{c}} u_{n}^{\delta} u_{n}^{p-\delta} \leq C R^{-\delta / 2} \int_{\mathbb{R}^{N}} u_{n}^{p-\delta} \leq C^{\prime} R^{-\delta / 2} .
$$

So, given $\varepsilon>0$, we can choose $R>0$ such that $\int_{B(0, R)^{c}} u_{n}^{p}<\varepsilon$ for every $n \in \mathbb{N}$. On the other hand, $u_{n} \rightarrow 0$ in $L^{p}(B(0, R))$, and this finishes the proof of compactness. 
By using dilatations, it is easy to see that $E$ is not included in $L^{p}\left(\mathbb{R}^{3}\right)$ for $p>6$. In order to deal with the case $p<18 / 7$, we prove the following

Claim: Given $p<18 / 7, M>0, T>0$, there exists a function $u \in E$ with compact support such that $\|u\|_{E} \leq 1, u=0$ in $B(0, M), \int_{\mathbb{R}^{3}}|u|^{p}>T$.

Indeed, for any $\varepsilon \in(0,1)$ define $R=\varepsilon^{-8 / 7}, S=\varepsilon^{-2 / 7}$ and:

$$
u_{\varepsilon}(r)= \begin{cases}0 & \text { if }|r-R| \geq S, \\ \varepsilon \frac{S-|r-R|}{S} & \text { if }|r-R|<S .\end{cases}
$$

We compute the norm of $u_{\varepsilon}$ in $E$ :

$$
\begin{gathered}
\int_{0}^{+\infty} u^{\prime}(r)^{2} r^{2} d r \leq \int_{R-S}^{R+S} \frac{\varepsilon^{2}}{S^{2}}(R+S)^{2} d r=2 \frac{\varepsilon^{2}}{S}(2 R)^{2} \leq 8, \\
\int_{0}^{+\infty} \int_{0}^{+\infty} u^{2}(r) u^{2}(s) r \operatorname{smin}\{r, s\} d r d s \leq \\
\int_{R-S}^{R+S} \int_{R-S}^{R+S} \varepsilon^{4}(R+S)^{3} d r d s \leq 4 S^{2} \varepsilon^{4}(2 R)^{3} \leq 32 .
\end{gathered}
$$

Moreover:

$$
\begin{gathered}
\int_{0}^{+\infty} u(r)^{p} r^{2} d r \geq \int_{R-S / 2}^{R+S / 2}\left(\frac{\varepsilon}{2}\right)^{p}(R-S / 2)^{2} d r \geq \\
S\left(\frac{\varepsilon}{2}\right)^{p}(R / 2)^{2}=\frac{1}{2^{p+2}} \varepsilon^{p} S R^{2}=\frac{1}{2^{p+2}} \varepsilon^{p-\frac{18}{7}} .
\end{gathered}
$$

Hence, the claim follows by taking $\varepsilon$ small enough (and dividing by a convenient constant).

Observe that the above claim readily implies that there is no continuous inclusion $E \subset L^{p}\left(\mathbb{R}^{3}\right)$ for $p<\frac{18}{7}$. We now show briefly that there is no inclusion at all, continuous or not. By the above claim, we can construct a sequence $u_{n}$ such that $\left\|u_{n}\right\|_{E} \leq 2^{-n}, \int_{\mathbb{R}^{3}}\left|u_{n}(x)\right|^{p} d x=1$ and with disjoint support. Observe that $v=\sum_{n=1}^{+\infty} u_{n} \in E$ and $\|v\|_{E} \leq 1$. Moreover, since $u_{n}$ have disjoint support, we have:

$$
\int_{\mathbb{R}^{3}}|v(x)|^{p} d x=\sum_{n=1}^{+\infty} \int_{\mathbb{R}^{3}}\left|u_{n}(x)\right|^{p} d x=+\infty .
$$

Remark 4.1. We conjecture that $E$ is not included in $L^{18 / 7}\left(\mathbb{R}^{3}\right)$.

Proof of Theorem 1.3, By Theorem [1.2, $J$ is well-defined, and it can be checked that $J$ is $C^{1}$ and that:

$$
J^{\prime}(u)(v)=\int_{\mathbb{R}^{3}} \nabla u \cdot \nabla v d x+\int_{\mathbb{R}^{3}} \int_{\mathbb{R}^{3}} \frac{u^{2}(x) u(y) v(y)}{|x-y|} d x d y-\int_{\mathbb{R}^{3}}|u|^{p-2} u v .
$$


Take $u \in E$, and define:

$$
M(u)=\int_{\mathbb{R}^{3}}|\nabla u|^{2} d x+\int_{\mathbb{R}^{3}} \int_{\mathbb{R}^{3}} \frac{u^{2}(x) u^{2}(y)}{|x-y|} d x d y .
$$

Define $\lambda=M(u)^{-1 / 3}$, and $v(x)=\lambda^{2} u(\lambda x)$. Observe that: $M(v)=$ $\lambda^{3} M(u)=1$, so, $\|v\|_{E} \leq \sqrt{2}$. By Theorem 1.2, there exists $C>0$ such that $\int_{\mathbb{R}^{3}}|v|^{p}<C$. Now observe that $\int_{\mathbb{R}^{3}}|u|^{p}=\lambda^{3-2 p} \int_{\mathbb{R}^{3}}|v|^{p}$. Summing up:

$$
J(u) \geq \frac{1}{4} M(u)-\frac{1}{p} \int_{\mathbb{R}^{3}}|u|^{p} d x \geq \frac{1}{4} M(u)-\frac{C}{p} M(u)^{\frac{2 p-3}{3}} \geq \frac{1}{8} M(u)-C^{\prime} .
$$

For last inequality just observe that the function $g(s)=\frac{s}{8}-\frac{C}{p} s^{\frac{2 p-3}{3}}$ is bounded below for $p<3$. Hence, $J$ is coercive.

By using the compactness of the inclusion $E \subset L^{p}\left(\mathbb{R}^{3}\right)$, it is easy to show that $I$ is weakly lower semicontinuous.

In order to finish the proof of Theorem 1.3 we just need to show that $\min J<0$. For that, fix $u \in E_{r}$, and define again $v_{\lambda}(x)=\lambda^{2} u(\lambda x)$. Hence:

$$
J\left(v_{\lambda}\right)=\frac{\lambda^{3}}{2} \int_{\mathbb{R}^{3}}|\nabla u|^{2} d x+\frac{\lambda^{3}}{4} \int_{\mathbb{R}^{3}} \int_{\mathbb{R}^{3}} \frac{u^{2}(x) u^{2}(y)}{|x-y|} d x d y-\frac{\lambda^{2 p-3}}{p} \int_{\mathbb{R}^{3}}|u|^{p} d x .
$$

So, for $\lambda$ small, $J\left(v_{\lambda}\right)$ takes negative values.

Proof of Theorem 1.4. By making the change of variables $\varepsilon=\lambda^{\frac{p-2}{4(3-p)}}$, $v(x)=\varepsilon^{\frac{2}{p-2}} u(\varepsilon x)$, problem (2) is equivalent to:

$$
-\Delta v+\varepsilon^{2} v+\left(v^{2} \star \frac{1}{|x|}\right) v=|v|^{p-2} v .
$$

Let us define the associated functional $J_{\varepsilon}: H_{r}^{1}\left(\mathbb{R}^{3}\right) \rightarrow \mathbb{R}$,

$$
J_{\varepsilon}(v)=\frac{1}{2} \int_{\mathbb{R}^{3}}\left(|\nabla v|^{2}+\varepsilon^{2}|v|^{2}\right) d x+\frac{1}{4} \int_{\mathbb{R}^{3}} \int_{\mathbb{R}^{3}} \frac{v^{2}(x) v^{2}(y)}{|x-y|} d x d y-\frac{1}{p} \int_{\mathbb{R}^{3}}|v|^{p} d x .
$$

Observe that $J_{\varepsilon}(v)=\varepsilon^{\frac{6-p}{p-2}} I_{\lambda}(u)$.

Take $u_{\lambda}$ minimizer of $I_{\lambda}$, and define $v_{\varepsilon}$ through the above change of variables: clearly, $v_{\varepsilon}$ is a minimizer for $J_{\varepsilon}$.

First, we claim that $\min J_{\varepsilon} \rightarrow \min J$ as $\varepsilon \rightarrow 0$. First, observe that $\min J_{\varepsilon}>\min J$. Take any $\delta>0$ and choose $v \in E$ such that $J(v)=$ $\min J$. Since $C_{0, r}^{\infty}\left(\mathbb{R}^{3}\right)$ is dense in $E_{r}$ (see Proposition 2.2), we can choose $\rho \in C_{0, r}^{\infty}\left(\mathbb{R}^{3}\right)$ such that $J(\rho)<\min J+\delta$. Hence, we can choose $\varepsilon_{0}>0$ such that for $\varepsilon \in\left(0, \varepsilon_{0}\right), J_{\varepsilon}(\rho)<\min J+2 \delta$. Since $\delta$ is arbitrary, we conclude the proof of the claim.

Hence, $\min J \leftarrow \min J_{\varepsilon}=J_{\varepsilon}\left(v_{\varepsilon}\right) \geq J\left(v_{\varepsilon}\right) \geq \min J$, so $v_{\varepsilon}$ is minimizing for $J$. Suppose, reasoning by contradiction, that there exists $c_{0}>0$ and $\varepsilon_{n} \rightarrow 0$ such that $d\left(v_{\varepsilon_{n}}, K\right)>c_{0}$. Since $J$ is coercive (Theorem[1.3), we have that $v_{\varepsilon_{n}}$ 
is bounded in $E$, and hence it converges weakly to some $v \in E$. Moreover, $J$ is weakly lower semicontinuous, so $v \in K$.

Recall that $J\left(v_{\varepsilon_{n}}\right) \rightarrow J(v)$, and, by Theorem [3.1, $\int_{\mathbb{R}^{3}}\left|v_{n}\right|^{p} \rightarrow \int_{\mathbb{R}^{3}}|v|^{p}$. From these facts we deduce that $\left\|v_{\varepsilon_{n}}\right\|_{E} \rightarrow\|v\|_{E}$. Since $E$ is uniformly convex, we conclude that $v_{\varepsilon_{n}} \rightarrow v$ strongly in $E$ (see [6], Proposition III.30). This is a contradiction with $d\left(v_{\varepsilon_{n}}, K\right)>c_{0}>0$.

\section{THE NONRADIAL CASE}

In this final section we consider the nonradial case, and we will prove Theorems 1.5, 1.6, 1.7.

We start with some estimates that will be of use later on. Given any $u \in C_{0}^{\infty}\left(\mathbb{R}^{3}\right)$ with support included in $B(0, M), e \in \mathbb{R}^{3}$ with $|e|=1$, and $N \in \mathbb{N}$, we define:

$$
u_{N}(x)=\sum_{i=1}^{N} u\left(x+i N^{2} e\right)
$$

Observe that $u_{N}$ is a sum of translations of $u$, and if $N^{2}>2 M$ the summands have disjoint support. In such case we have:

$$
\begin{gathered}
\int_{\mathbb{R}^{3}}\left|\nabla u_{N}\right|^{2} d x=N \int_{\mathbb{R}^{3}}|\nabla u|^{2} d x, \\
\int_{\mathbb{R}^{3}}\left|u_{N}\right|^{p} d x=N \int_{\mathbb{R}^{3}}|u|^{p} d x,
\end{gathered}
$$

$\int_{\mathbb{R}^{3}} \int_{\mathbb{R}^{3}} \frac{u_{N}^{2}(x) u_{N}^{2}(y)}{|x-y|} d x d y=\sum_{i, j=1}^{N} \int_{\mathbb{R}^{3}} \int_{\mathbb{R}^{3}} \frac{u^{2}\left(x+i N^{2} e\right) u^{2}\left(y+j N^{2} e\right)}{|x-y|} d x d y=$ $N \int_{\mathbb{R}^{3}} \int_{\mathbb{R}^{3}} \frac{u^{2}(x) u^{2}(y)}{|x-y|} d x d y+\sum_{i \neq j}^{N} \int_{\mathbb{R}^{3}} \int_{\mathbb{R}^{3}} \frac{u^{2}\left(x+i N^{2} e\right) u_{N}^{2}\left(y+j N^{2} e\right)}{|x-y|} d x d y$.

Now we compute:

$$
\begin{gathered}
\sum_{i \neq j}^{N} \int_{\mathbb{R}^{3}} \int_{\mathbb{R}^{3}} \frac{u^{2}\left(x+i N^{2} e\right) u^{2}\left(y+j N^{2} e\right)}{|x-y|} d x d y= \\
\sum_{i \neq j}^{N} \int_{B(0, M)} \int_{B(0, M)} \frac{u^{2}(x) u^{2}(y)}{\left|x-y+(j-i) N^{2} e\right|} d x d y \leq \\
\sum_{i \neq j}^{N} \int_{B(0, M)} \int_{B(0, M)} \frac{u^{2}(x) u^{2}(y)}{N^{2}-2 M} d x d y=\frac{N^{2}-N}{N^{2}-2 M}\left(\int_{\mathbb{R}^{3}} u^{2}(x) d x\right)^{2} .
\end{gathered}
$$

So, we get: 


$$
\left|\int_{\mathbb{R}^{3}} \int_{\mathbb{R}^{3}} \frac{u_{N}^{2}(x) u_{N}^{2}(y)}{|x-y|} d x d y-N \int_{\mathbb{R}^{3}} \int_{\mathbb{R}^{3}} \frac{u^{2}(x) u^{2}(y)}{|x-y|} d x d y\right| \leq C,
$$

where $C>0$ is a constant depending on the original function $u$ and is independent of $N$.

Proof of Theorem 1.5. First, we show that $E \subset L^{3}\left(\mathbb{R}^{3}\right)$. Actually, this is well-known from [21. Recall that $-\Delta \phi=u^{2}$, and $\phi \in D^{1,2}\left(\mathbb{R}^{3}\right)$. By multiplying by $|u|$ and integrating, we obtain:

$$
\int_{\mathbb{R}^{3}}|u|^{3}=\int_{\mathbb{R}^{3}}\langle\nabla \phi, \nabla|u|\rangle \leq \frac{1}{2} \int_{\mathbb{R}^{3}}|\nabla u|^{2}+|\nabla \phi|^{2} d x .
$$

Recall now that:

$$
\int_{\mathbb{R}^{3}}\left|\nabla \phi_{u}(x)\right|^{2} d x=\int_{\mathbb{R}^{3}} \int_{\mathbb{R}^{3}} \frac{u^{2}(x) u^{2}(y)}{4 \pi|x-y|} d x d y .
$$

This implies that $E \subset L^{3}\left(\mathbb{R}^{3}\right)$. Moreover, $E \subset D^{1,2}\left(\mathbb{R}^{3}\right) \subset L^{6}\left(\mathbb{R}^{3}\right)$ by Sobolev embedding. By interpolation we conclude that $E \subset L^{p}\left(\mathbb{R}^{3}\right)$ for all $p \in[3,6]$.

Let us now show that $E$ is not included in any $L^{p}$ space for $p<3$. Fix $u \in C_{0}^{\infty}\left(\mathbb{R}^{3}\right)$; for $N \in \mathbb{N}$ define $u_{N}$ as in (11). Take also $\lambda_{N}=N^{-1 / 3}$, and define $v_{N}=\lambda_{N}^{2} u_{N}\left(\lambda_{N} x\right)$. By using (12), (13), (14), we obtain:

$$
\begin{gathered}
\int_{\mathbb{R}^{3}}\left|\nabla v_{N}\right|^{2} d x=\lambda_{N}^{3} \int_{\mathbb{R}^{3}}\left|\nabla u_{N}\right|^{2}=C_{1} . \\
\int_{\mathbb{R}^{3}} \int_{\mathbb{R}^{3}} \frac{v_{N}^{2}(x) v_{N}^{2}(y)}{|x-y|} d x d y=\lambda_{N}^{3} \int_{\mathbb{R}^{3}} \int_{\mathbb{R}^{3}} \frac{u_{N}^{2}(x) u_{N}^{2}(y)}{|x-y|} d x d y \\
\leq \lambda_{N}^{3}\left(C_{2} N+C_{3}\right) \leq C_{4} . \\
\int_{\mathbb{R}^{3}}\left|v_{N}\right|^{p} d x=\lambda_{N}^{2 p-3} \int_{\mathbb{R}^{3}}\left|u_{N}\right|^{p} d x=C_{5} \lambda_{N}^{2 p-3} N .
\end{gathered}
$$

Above, $C_{k}$ are positive constants that depend upon $u$, but are independent of $N$. So, $\left\{v_{N}\right\}$ is a bounded sequence in $E$ such that $\int\left|v_{N}\right|^{p} d x \rightarrow+\infty$ if $p<3$.

This already implies that there is no continuous inclusion from $E$ in $L^{p}\left(\mathbb{R}^{3}\right)$. Now, we can argue as in the proof of Theorem 1.2 , to conclude that $E$ is not included in $L^{p}$.

We now turn our attention to Theorem 1.6. First of all, we remind a result of [25] (Theorem 4.3):

Proposition 5.1. For any $\lambda>0$ and $p \in(2,3),\left.I_{\lambda}\right|_{H_{r}^{1}\left(\mathbb{R}^{3}\right)}$ is coercive and weak lower semicontinuous. In particular, it has a minimum. 
As we see, Theorem 1.6 is in contrast with the above result.

To start with, let us consider the map $m:[0,+\infty) \rightarrow[-\infty,+\infty), m(\lambda)=$ $\inf I_{\lambda}$. It is easy to check that $m$ is nondecreasing and upper semicontinuous, and $m(0)=-\infty$.

Moreover, $m_{\lambda} \leq I_{\lambda}(0)=0$ for any $\lambda \in \mathbb{R}$. Actually, in 25] it is proved that $m_{\lambda}=0$ for $\lambda$ large and $p \in(2,3)$. Let us reproduce the proof here, for the sake of completeness:

$$
\begin{aligned}
I_{\lambda}(u)= & \frac{1}{2} \int_{\mathbb{R}^{3}}\left(|\nabla u|^{2}+u^{2}\right) d x+\frac{\lambda}{4} \int_{\mathbb{R}^{3}} \int_{\mathbb{R}^{3}} \frac{u^{2}(x) u^{2}(y)}{|x-y|} d x d y-\frac{1}{p} \int_{\mathbb{R}^{3}}|u|^{p} d x= \\
& \frac{1}{2} \int_{\mathbb{R}^{3}}\left(|\nabla u|^{2}+u^{2}\right) d x+\pi \lambda \int_{\mathbb{R}^{3}}\left|\nabla \phi_{u}\right|^{2} d x-\frac{1}{p} \int_{\mathbb{R}^{3}}|u|^{p} d x .
\end{aligned}
$$

Then, if $\lambda \pi \geq \frac{1}{2}$, we can use inequality (15) to conclude:

$$
I_{\lambda}(u) \geq \int_{\mathbb{R}^{3}}\left(\frac{1}{2} u^{2}+|u|^{3}-\frac{1}{p}|u|^{p}\right) d x \geq 0 .
$$

Proof of Theorem 1.6. With all these preliminaries, Theorem 1.6 follows from the following

Claim: If $m_{\lambda}<0$, then $m_{\lambda}=-\infty$.

If $m_{\lambda}<0$, we can use density of $C_{0}^{\infty}\left(\mathbb{R}^{3}\right)$ in $H^{1}\left(\mathbb{R}^{3}\right)$ to find $u \in C_{0}^{\infty}\left(\mathbb{R}^{3}\right)$ such that $I_{\lambda}(u)<0$. Given such function $u$, define $u_{N}$ as in the beginning of the section. By (12), (13), (14),

$$
I_{\lambda}\left(u_{N}\right) \leq N I_{\lambda}(u)+C
$$

where $C>0$ is independent of $N$. So, we conclude that $\lim _{N \rightarrow+\infty} I_{\lambda}\left(u_{N}\right)=$ $-\infty$.

As we have seen, Theorems 1.5 and1.6 make clear the differences between the radial and nonradial cases. This phenomenon is due to the nonlocal term given by the Coulomb energy; observe that this term increases when we make Schwartz rearrangements.

In order to obtain more consequences from this, let us consider the problem:

$$
\begin{array}{ll}
-\Delta u+u+\lambda\left(u^{2} \star \frac{1}{|x|}\right) u=|u|^{p-2} u, & \text { in } B(0, R) \\
u(x)=0 & \text { in } \partial B(0, R) .
\end{array}
$$

As always, $p \in(2,3)$. The associated energy functional is nothing but $\left.I_{\lambda}\right|_{H_{0}^{1}(B(0, R))}$ (in the radial case, $\left.\left.I_{\lambda}\right|_{H_{0, r}^{1}(B(0, R))}\right)$. Define:

$$
m(R, \lambda)=\left.\inf I_{\lambda}\right|_{H_{0}^{1}(B(0, R))}, \quad \bar{m}(R, \lambda)=\left.\inf I_{\lambda}\right|_{H_{0, r}^{1}(B(0, R))} .
$$

Lemma 5.2. The infima that define $m$ and $\bar{m}$ are achieved. 
Proof. The proof follows from [25]. Indeed, as in (15),

$$
c_{\lambda} \int_{B(0, R)}|u|^{3}=c_{\lambda} \int_{B(0, R)}\langle\nabla \phi, \nabla|u|\rangle \leq \frac{1}{4} \int_{B(0, R)}|\nabla u|^{2}+\frac{\lambda}{4} \int_{\mathbb{R}^{3}}|\nabla \phi|^{2},
$$

where $c_{\lambda}=\frac{\sqrt{\lambda}}{2}>0$. Let us point out that $\phi=\frac{1}{4 \pi|x|} \star u^{2}$, so $-\Delta \phi=u^{2}$ in $\mathbb{R}^{N}$, so $\phi$ is not equal to zero on the boundary.

We have:

$$
I_{\lambda}(u) \geq \int_{B(0, R)}\left(\frac{1}{4}|\nabla u|^{2}+\frac{1}{2} u^{2}+c_{\lambda}|u|^{3}-\frac{1}{p+1}|u|^{p+1}\right) d x \geq \int_{B(0, R)} \frac{1}{4}|\nabla u|^{2} d x-C .
$$

Therefore, $\left.I_{\lambda}\right|_{H_{0}^{1}(B(0, R))}$ is coercive. It is not difficult to check that it is also weak lower semicontinuous, so the existence of a minimum holds. The same arguments work in the radial case.

\section{Proof of Theorem 1.7.}

(1) Suppose that $p \in(2,3), \lambda \in\left(0, \lambda_{0}\right)$, where $\lambda_{0}$ is given in Theorem 1.6 .

It suffices to show that for $R$ large, $m(R, \lambda)<\bar{m}(R, \lambda)$. Have in mind Theorem 1.6 and Proposition 5.1, so:

$$
\lim _{R \rightarrow+\infty} m(R, \lambda)=\inf I_{\lambda}=-\infty, \quad \lim _{R \rightarrow+\infty} \bar{m}(R, \lambda)=\left.\inf I_{\lambda}\right|_{H_{r}^{1}\left(\mathbb{R}^{3}\right)}>-\infty .
$$

Then, for $R$ large, $m(R, \lambda)<\bar{m}(R, \lambda)$.

(2) We now assume $p \in(18 / 7,3)$ and $R$ fixed.

This case does not follow as above, $\operatorname{since} \lim _{\lambda \rightarrow 0} m(R, \lambda)=\lim _{\lambda \rightarrow 0} \bar{m}(R, \lambda)=$ $-\infty$.

We make again the change of variables: $v(x)=\varepsilon^{\frac{2}{p-2}} u(\varepsilon x), \varepsilon=\lambda^{\frac{p-2}{4(3-p)}}$, to arrive to the problem:

$$
\begin{array}{ll}
-\Delta v+\varepsilon^{2} v+\left(v^{2} \star \frac{1}{|x|}\right) v=|v|^{p-2} v, & \text { in } B(0, R / \varepsilon) \\
v(x)=0 & \text { in } \partial B(0, R / \varepsilon) .
\end{array}
$$

As in the proof of Theorem 1.4, define

$J_{\varepsilon}(v)=\frac{1}{2} \int_{\mathbb{R}^{3}}\left(|\nabla v|^{2}+\varepsilon^{2}|v|^{2}\right) d x+\frac{1}{4} \int_{\mathbb{R}^{3}} \int_{\mathbb{R}^{3}} \frac{v^{2}(x) v^{2}(y)}{|x-y|} d x d y-\frac{1}{p} \int_{\mathbb{R}^{3}}|v|^{p} d x$.

Define $c(\varepsilon)=\left.\inf J_{\varepsilon}\right|_{H_{0}^{1}(B(0, R / \varepsilon))}, \bar{c}(\varepsilon)=\left.\inf J_{\varepsilon}\right|_{H_{0, r}^{1}(B(0, R / \varepsilon))}$. Since $J_{\varepsilon}(v)=$ $\varepsilon^{\frac{6-p}{p-2}} I_{\lambda}(u)$, we have that:

$$
c(\varepsilon)=\varepsilon^{\frac{6-p}{p-2}} m(\lambda, R), \quad \bar{c}(\varepsilon)=\varepsilon^{\frac{6-p}{p-2}} \bar{m}(\lambda, R) .
$$

Observe now that $\bar{c}(\varepsilon) \geq\left.\inf J_{0}\right|_{E}>-\infty$ (Theorem 1.3). We now claim that $\lim _{\varepsilon \rightarrow 0} c(\varepsilon)=-\infty$, and this finishes the proof. 
Take $M>0$ arbitrary; by Theorem 1.5, $E$ is not included in $L^{p}\left(\mathbb{R}^{3}\right)$, and there exists $u \in C_{0}^{\infty}\left(\mathbb{R}^{3}\right)$ such that $\|u\|_{E} \leq 1, \int_{\mathbb{R}^{3}}|u|^{p}>M$. Now choose $\varepsilon>0$ small enough such that:

$$
\text { supp } u \subset B(0, R / \varepsilon) \text { and } \varepsilon^{2} \int_{\mathbb{R}^{3}}|u|^{2} \leq 1 \text {. }
$$

In such case, $u \in H_{0}^{1}(B(0, R / \varepsilon))$ and $c(\varepsilon) \leq J_{\varepsilon}(u) \leq 3-M$.

Remark 5.3. Being u a nonradial minimizer of $\left.I_{\lambda}\right|_{H_{0}^{1}(B(0, R))}$, we have a family of minimizers $\{u \circ g: g \in O(N)\}$. This implies that the minimum is degenerate, and hence it does not satisfy the conditions of [16] for orbital stability. Let us denote by $K$ the set of minimizers of $\left.I_{\lambda}\right|_{H_{0}^{1}(B(0, R))}$; then, $K$ is orbitally stable in the sense of [10].

Remark 5.4. In the above result we have emphasized the breaking of symmetry of the minimizer: let us now consider briefly the multiplicity of positive solutions.

Under the conditions of Theorem 1.7 we actually obtain the existence of two solutions, a minimizer for $m(R, \lambda)$ and a different one for $\bar{m}(R, \lambda)$. Moreover, $m(R, \lambda)$ is negative and by taking $\lambda$ smaller (if necessary), $\bar{m}(R, \lambda)$ is also negative. So, both minima are negative, and hence they yield two positive nontrivial solutions.

Even more, observe that 0 is a local minimum of $\left.I_{\lambda}\right|_{H^{1}\left(\mathbb{R}^{3}\right)}$. It is not difficult to show that the Palais-Smale condition holds (remember that $I_{\lambda}$ is coercive). The well-known mountain-pass theorem ([1]) implies the existence of a third nontrivial solution.

Everything said above can be applied to the functional:

$I_{\lambda}^{+}(u)=\frac{1}{2} \int_{\mathbb{R}^{3}}\left(|\nabla u|^{2}+u^{2}\right) d x+\frac{\lambda}{4} \int_{\mathbb{R}^{3}} \int_{\mathbb{R}^{3}} \frac{u^{2}(x) u^{2}(y)}{|x-y|} d x d y-\frac{1}{p} \int_{\mathbb{R}^{3}}\left(u^{+}\right)^{p} d x$.

By the maximum principle, we obtain the existence of three positive solutions.

Aknowledgement: The author thanks Prof. Ireneo Peral for many discussions on these problems during a stay in the Universidad Autónoma of Madrid, as well as for the warm hospitality.

\section{REFERENCES}

[1] A. Ambrosetti and P. H. Rabinowitz, Dual variational methods in critical point theory and applications, J. Funct. Anal. 14 (1973), 349-381.

[2] A. Ambrosetti and D. Ruiz, Multiple bound states for the Schrödinger-Poisson problem, to appear in Comm. Contemp. Math.

[3] V. Benci and D. Fortunato, An eigenvalue problem for the Schrödinger-Maxwell equations, Top. Meth. Nonl. Anal. 11 (1998), 283-293.

[4] O. Bokanowski and N.J. Mauser, Local approximation of the Hartree-Fock exchange potential: a deformation approach, $\mathrm{M}^{3} \mathrm{AS} 9$ (1999), 941-961. 
[5] O. Bokanowski, J.L. López and J. Soler, On an exchange interaction model for the quantum transport; the Schrödinger-Poisson-Slater term, M $^{3} \mathrm{AS} 13$ (2003), 13971412.

[6] H. Brezis, Analyse fonctionelle, Théorie et applications, Masson Ed., Paris, 1983.

[7] H. Beresticky and P. L. Lions, Nonlinear Scalar field equations I: Existence of a ground state, Arch. Rat. Mech. Anal 82 (1983), 313-345.

[8] I. Catto, C. Le Bris and P. L. Lions, On some periodic Hartree-type models for crystals, Ann. Inst. H. Poincaré Anal. Non Linéaire 19 (2002), 143-190.

[9] I. Catto, C. Le Bris, and P. L. Lions, On the thermodynamic limit for Hartree-Fock type models, Ann. Inst. H. Poincaré Anal. Non Linéaire 18 (2001), 687-760.

[10] T. Cazenave and P. L. Lions, Orbital stability of standing waves for some nonlinear Schrödinger equations, Comm. Math. Phys. 85 (1982), 549-561.

[11] T. D'Aprile and D. Mugnai, Solitary waves for nonlinear Klein-Gordon-Maxwell and Schrödinger-Maxwell equations, Proc. Royal Soc. Edinburgh 134A (2004), 893-906.

[12] T. D'Aprile and D. Mugnai, Non-existence results for the coupled Klein-GordonMaxwell equations, Adv. in Nonl. Studies 4 (2004), 307-322.

[13] T. D'Aprile and J. Wei, On bound states concentrating on spheres for the MaxwellSchrödinger equation, SIAM J. Math. Anal. 37 (2005), 321-342.

[14] T. D'Aprile and J. Wei, Standing waves in the Maxwell-Schrödinger equation and an optimal configuration problem, Calc. Var. 25 (2005), 105-137.

[15] B. Gidas, W.-M. Ni and L. Nirenberg, Symmetry and related properties via the maximum principle, Comm. Math. Phys. 68 (1979), 209-243.

[16] M. Grillakis, J. Shatah and W. Strauss, Stability theory of solitary waves in the presence of symmetry I y II, J. Funct. Anal. 74 (1987), 160-197 y 94 (1990), 308-348.

[17] I. Ianni and G. Vaira, Semiclassical states for the Schrödinger-Poisson problem with an external potential and a density charge: concentration around a sphere, preprint.

[18] H. Kikuchi, On the existence of a solution for elliptic system related to the MaxwellSchrödinger equations, Nonlinear Anal. 67 (2007), no. 5, 1445-1456.

[19] H. Kikuchi, Existence and orbital stability of standing waves for nonlinear Schrödinger equations via the variational method, Doctoral Thesis.

[20] E. H. Lieb and M. Loss, Analysis, Graduate Studies in Mathematics, vol. 14, AMS, 1997.

[21] P.-L. Lions, Solutions of Hartree-Fock equations for Coulomb systems, Comm. Math. Physics 109 (1984), 33-97.

[22] N.J. Mauser, The Schrödinger-Poisson-X $\alpha$ equation, Applied Math. Letters 14 (2001), 759-763.

[23] L. Pisani and G. Siciliano, Neumann condition in the Schrödinger-Maxwell system, Topol. Methods Nonlinear Anal. 29 (2007), 251-264.

[24] D. Ruiz, Semiclassical states for coupled Schrodinger-Maxwell equations: concentration around a sphere, $\mathrm{M}^{3} \mathrm{AS} 15$ (2005), 141-164.

[25] D. Ruiz, The Schrödinger-Poisson equation under the effect of a nonlinear local term, J. Funct. Anal. 237 (2006), 655-674.

[26] O. Sánchez and J. Soler, Long-time dynamics of the Schrödinger-Poisson-Slater system, J. Statistical Physics 114 (2004), 179-204.

[27] J.C. Slater, A simplification of the Hartree-Fock method, Phys. Review 81 (1951), 385-390.

[28] D. Smets, J. Su and M. Willem, Non-radial ground states for the Hénon equation, Commun. Contemp. Math. 4 (2002), 467-480.

[29] J. Su, Z.-Q. Wang and M. Willem, Nonlinear Schrödinger equations with unbounded and decaying radial potentials, Commun. Contemp. Math. 9 (2007), 571-583.

[30] J. Su, Z.-Q. Wang and M. Willem, Weighted Sobolev embedding with unbounded and decaying radial potentials, J. Differential Equations 238 (2007), 201-219. 
[31] Zhengping Wang and Huan-Song Zhou, Positive solution for a nonlinear stationary Schrödinger-Poisson system in $\mathbb{R}^{3}$, Discrete and Continuous Dynamical Systems, 18 (2007), 809-816.

Dpto. Análisis Matemático, Universidad de Granada, 18071 Granada, Spain

E-mail address: daruiz@ugr.es 